\title{
Mental Health Interventions for Parent Carers of Children with Autistic Spectrum Disorder: Practice Guidelines from a Critical Interpretive Synthesis (CIS) Systematic Review
}

\author{
Denise Catalano $^{1, *(1)}$, Linda Holloway ${ }^{1(1)}$ and Elias Mpofu ${ }^{1,2,3}$ \\ 1 Department of Rehabilitation and Health Services, University of North Texas, Denton, TX 76203, USA; \\ Linda.Holloway@unt.edu (L.H.); Elias.Mpofu@unt.edu (E.M.) \\ 2 Department of Educational Psychology, University of Johannesburg, Johannesburg, \\ Auckland Park 2006, South Africa \\ 3 Department of Rehabilitation Counselling, University of Sydney, Lidcombe, NSW 2141, Australia \\ * Correspondence: denise.catalano@unt.edu; Tel.: +1-940-891-6893
}

Received: 20 December 2017; Accepted: 10 February 2018; Published: 14 February 2018

\begin{abstract}
Parent carers of children with Autism Spectrum Disorder (ASD) often report increased levels of stress, depression, and anxiety. Unmet parent carer mental health needs pose a significant risk to the psychological, physical, and social well-being of the parents of the child affected by ASD and jeopardize the adaptive functioning of the family as well as the potential of the child affected by ASD. This systematic review identifies key qualities of interventions supporting the mental health of parent carers and proposes practitioner-parent carer support guidelines. A search of four databases (Medline, PubMed, PsycINFO, and Social Science Data) was conducted to identify studies that met the following criteria: (1) an intervention was delivered to parent carers of a child with ASD under the age of 18 years; (2) the research design allowed for a comparison on outcomes across groups; and (3) outcome measures of the parent carers' mental health were used. A total of 23 studies met the inclusion criteria. A critical interpretive synthesis approach was used to produce an integrated conceptualization of the evidence. Findings suggest practitioner guidelines to support the mental health and wellbeing of parent carers should include addressing the parent's self-perspective taking and skill for real time problem-solving.
\end{abstract}

Keywords: autism; autism spectrum disorder; parent carer; mental health; stress; intervention; systematic review

\section{Introduction}

Parents who are primary carers of a child affected with Autism Spectrum Disorder (ASD) are often found to experience higher levels of stress and poorer physical health when compared with parents of children of typical development [1-3], parents of children diagnosed with other disabilities [4,5], or when compared to the general population [6-11]. The parenting stress experienced by parents of a child affected with ASD therefore appears to pose a greater risk to the parents' psychological and health-related quality of life. ASD is a neurodevelopmental disorder characterized by deficits in social interactions and communication skills, both verbal and non-verbal, restricted interests, and stereotypical behaviors [12]. The manifestation of ASD symptoms may range from mild to severe and vary from individual to individual. It is estimated that 1 in 160 children worldwide (or 62.5 per 10,000) are identified with ASD [13], with Japan reported to have the highest prevalence of approximately 161 children per 10,000 identified with the disorder [14]. In the United States, the number 
of children identified with ASD has increased from 1 in 150 (or 66.7 per 10,000) children estimated in the year 2000, to 1 in 68 (or 147 per 10,000) in 2016, an increase of 119.4 percent [15].

Carers of children diagnosed with ASD, often referred to as "autism", are generally the parents of the child [16]. These parents are at high risk for "caregiver syndrome" or "caregiver stress", a condition of exhaustion, anger, rage, or guilt that results from unrelieved caring for a chronically ill dependent [17]. "Caregiver burnout" and "caregiver burden" are additional terms often used to describe a state of physical, emotional, and mental exhaustion that may be accompanied by a change in attitude, from positive and caring to negative and unconcerned, as a consequence of attending to the ongoing demands inherent in caring for a dependent individual [18,19].

Some parents become overwhelmed by the daily hassles and general life stresses they experience parenting a child affected by ASD [20]. A growing body of evidence suggests that parents of a child affected with ASD experience higher rates of depression and anxiety [21-27], fatigue [28], increased problems with physical health and bodily pain [29], and poorer overall well-being [24] and quality of life $[26,30]$. Increased parenting stress is attributed to the need to provide constant supervision and assistance to the daily living skills of the child, ongoing sleep disruption, lack of available respite care, and lack of responsiveness by school personnel and related services [31-34]. Moreover, the behavior of children affected with ASD may be difficult to manage due to behavioral anomalies, including temper tantrums and aggressive, self-abusive, destructive, obsessive, ritualistic, impulsive, and self-stimulating behaviors that can pose potential physical harm to the parent carer as well as siblings or family members and friends $[8,26,35]$. There is a high likelihood that a child affected by ASD may also be affected by a comorbid condition, such as specific phobia (SP), obsessive-compulsive disorder (OCD), or attention-deficit hyperactivity (ADHD), adding to difficulties for parent carers tasked with trying to manage the child's behavior [36]. Externalizing behaviors (e.g., hyperactivity and conduct problems) have consistently been found to explain a significant portion of parental distress and poor physical health [37].

Addressing the unmet mental health needs of parent carers with a child affected by ASD is necessary in order to improve the overall health and quality of life of the parents, as well as those of other family members [38]. Moreover, improving the parents' mental health and wellbeing enhances the potential of the child to also achieve a better quality of life. Improvements to the child's overall behavior and functioning is facilitated when behavioral treatment interventions being delivered to the child are supplemented by continued implementation by the parent carers in the home environment [39]. The effectiveness of the child's behavioral treatment interventions have been reported to be diminished when parents' mental health needs are unmet [40].

The health-related quality of life of parent carers of a child affected by ASD may be enhanced by directly reducing parental stress through the engagement of parent carers in effective mental health support strategies. Practice guidelines for supporting the mental health wellbeing of parent carers of children with ASD are important for translating public health care services received by their children into real, sustainable health benefits for them, their families, and their community. Currently guidelines to support the mental health of parents who have a child affected by ASD do not exist, yet these guidelines would be important to identify in order to promote the delivery of cost-effective services to parents who need assistance. Identifying evidence-supported parent carer mental health interventions can provide practitioners, and their parent carer partners, with working guidelines for delivering mental health support services that, upon further development, could be adopted by state and federal agencies with the responsibility for providing services to children affected by ASD and their families and parents.

\section{Objectives of Study}

We specifically aimed to identify and critically cross-walk findings from the extant studies with the intention to propose practice guidelines for practitioners and parent carers for improving the mental health and psychological wellbeing of those parents who have a child affected by ASD and 
are challenged with maintaining their own wellbeing. Our goal was to apply a critical integrative synthesis analysis to collate the emerging evidence from a diverse body of literature examining mental health supports for parent carers of a child affected by ASD. In sum, we synthesized the nature and results of mental health intervention studies delivered to parent carers of a child affected by ASD reported in studies that examined the effectiveness of mental health interventions as well as qualitative reports in order to provide health and allied-health professionals with practice guidelines that could be implemented into a standard practice of care to address the mental health needs of parent carers of a child affected by ASD.

\section{Method}

\subsection{Critical Interpretative Synthesis}

Critical Interpretive Synthesis (CIS) is a systematic review that allows for triangulation of the evidence gathered from effectiveness (i.e., quantitative) and qualitative studies into new conceptual constructs, grounded in evidence and resulting from a critical interpretation of that evidence [41,42]. The synthesis results in the development of synthetic constructs, transforming the underlying evidence into new conceptual constructs and allow for various aspects of an identified phenomenon described in a diverse body of evidence to be expressed in a more comprehensive and useful way. These synthetic constructs, formed from the critical integration of findings across all studies included in the review, can then be organized into a coherent explanatory theoretical framework [42]. A CIS review of the broad and complex literature that intersects parenting, mental health, and ASD can provide more useful and pragmatic practice guidelines for practitioners and parent carers than what can be produced from conventional reviews alone (e.g., meta-analyses).

\subsection{Search Criteria and Literature Search}

The focus of the synthesis reported in this review was on interventions provided to parents of a child with ASD that resulted in the parent carers improved mental health. Studies included in this review met the following inclusion criteria: (1) an intervention was delivered directly to one or both parent carers of a child with ASD under the age of 18 years; (2) the study involved a research design allowing for a comparison across groups on the outcome of an intervention; (3) an outcome measure of parental mental health was used; and (4) the study was published in English in a peer-reviewed journal. Parent carers were defined as maternal mothers or paternal fathers who resided in the same home as their child with ASD and who had responsibility for the child's care. "Mental health" was defined in accordance with the World Health Organization definition of mental health—"a state of well-being in which every individual realizes his or her own potential, can cope with the normal stresses of life, can work productively and fruitfully, and is able to make a contribution to her or his community" [43] (para. 1). Outcome measures included any instrument that assessed some aspect of mental health, including measures of stress, anxiety, depression, quality of life (including health-related quality of life), and subjective well-being. There were no publication date restrictions applied, and other systematic reviews or review-based articles were not included.

\subsection{Search Procedure}

A systematic search of the databases Medline, PubMed, PsycINFO, and Social Science Data was conducted using key search terms identified from relevant papers found in a preliminary search, and from a search of relevant Medical Subject Headings (MeSH) terms which were then incorporated into an expanded search strategy. The MeSH and text words for Autism (Autism, Autism Spectrum Disorder, ASD, developmental disorder, Asperger's Syndrome, Asperger's) were combined with $\mathrm{MeSH}$ and text terms for parent carers (mother, father, caregiver, carer, maternal, paternal), MeSH and text words for intervention (intervention, support, effects, improve, train, treatment, program), and $\mathrm{MeSH}$ and text words for mental health (mental health, mental wellbeing, psychological wellbeing, 
wellbeing, stress, depression, anxiety). Boolean "OR" was used to connect search terms within each set of text words, while "AND" was used to connect the four sets of text words. Terms that had plural forms (e.g., parents) were truncated (i.e., "**") and hyphenated words (e.g., well-being) were entered in both their hyphenated and non-hyphenated form. Since both quantitative and qualitative studies were desired, no qualifier was entered for research design.

The search was conducted from 15 May through 15 July 2017 by the primary author. Citations identified by the search were exported to the first author's RefWorks account for de-duplication. The first and second co-authors assessed remaining studies for relevance by screening titles and abstracts, discarding those that did not meet the inclusion criteria. Full-text versions of the retained studies were obtained and examined independently by the first and second co-authors for study eligibility. Reference lists of the retained articles were hand searched for potential additional articles. Additional articles found were reviewed in their full-text version by the first two-co-authors to ensure they met the eligibility standards. Inter-observer agreement of the retained studies was $100 \%$.

\subsection{Quality Appraisal}

A quality appraisal of each qualitative study was conducted prior to the study being included in the final review and synthesis. The quality of a qualitative study is largely determined by the quality of the published report since there is no consensus on a set of specific standards [44]. The individual studies included in the present integrative synthesis were assessed using a published checklist [45] that identifies nine specific elements of the published report, each evaluated using a four-point Likert-type scale $(4=$ good, $3=$ fair, $2=$ poor, $1=$ very poor). The nine elements included: (1) the abstract and title, (2) introduction and aims, (3) method and data, (4) sampling procedure, (5) data analysis, (6) ethics and bias, (7) findings and results, (8) transferability/generalizability, and (9) implications and usefulness. Scores for each article are summed with higher scores reflecting higher methodological quality (scores range from 9 (very poor) to 36 (very good). The first author assessed the quality of each study retained in the search and found the majority of the studies were of good methodological quality, with scores ranging from 24 to 35 . The 2nd co-author randomly selected $30 \%$ of the studies and conducted an independent assessment. No significant disagreements occurred (scores between co-authors differentiated by no more than one or two points on average) and discrepancies were discussed until consensus was achieved. Both co-authors agreed that one particular study should be excluded based on having a score substantially lower than the other studies (score $=14$ ) due to several noted and significant methodological limitations.

\section{Results}

\subsection{Search Results}

Figure 1 provides the number of total identified citations and results of the eligibility screening process. The search yielded 2361 citations, with 942 citations determined to be duplicates. Of the remaining 1419 records, 1367 records were deemed ineligible following a review of the title and abstract information. Full-text articles were obtained of the remaining 52 studies and reviewed independently by both the first and second co-authors. A total of 29 studies were excluded by mutual consensus of the co-authors (see explanations provided in Figure 1) resulting in a total of 23 studies included in the synthesis. 


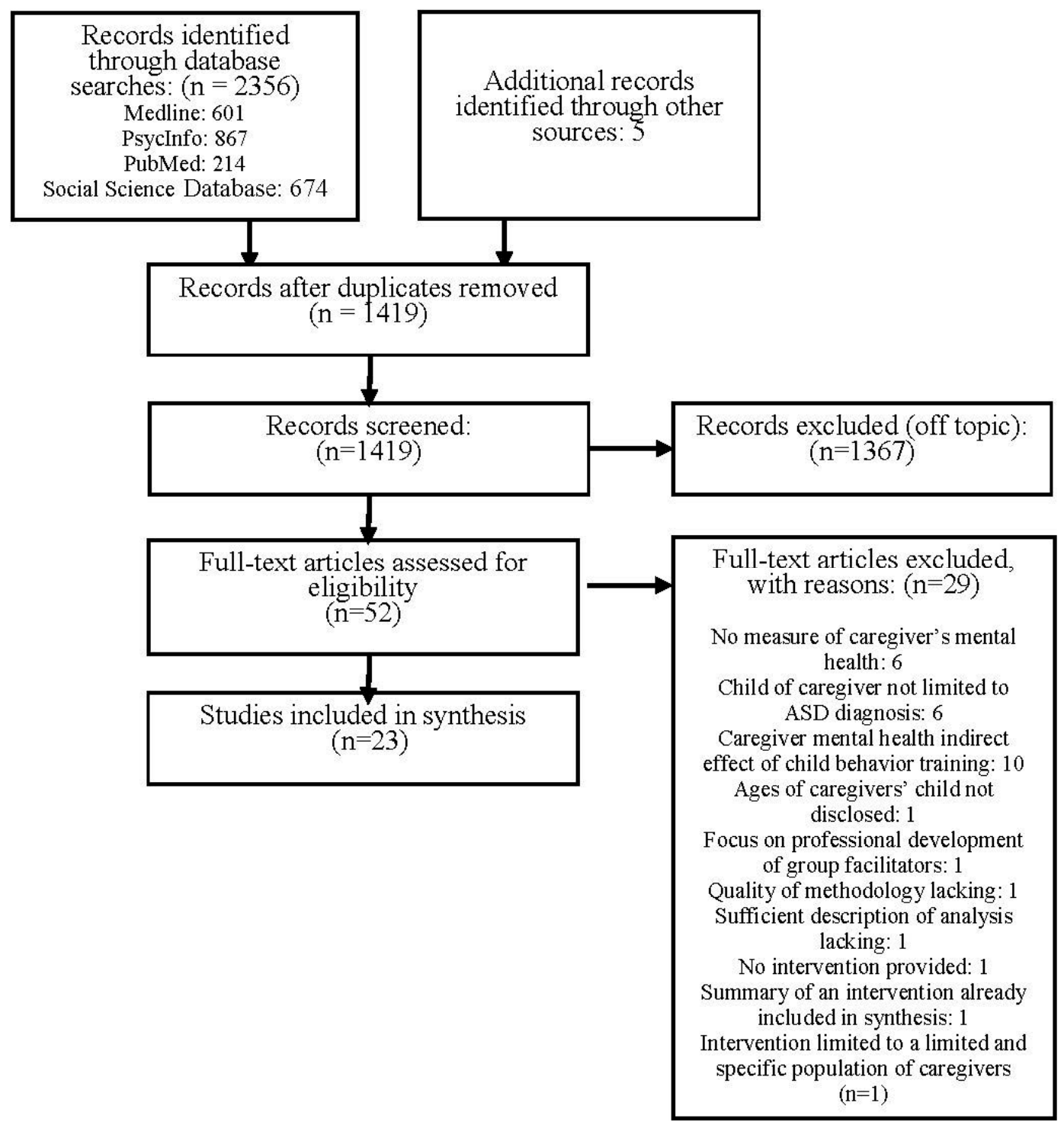

Figure 1. Flowchart of study selection process.

\subsection{Study Characteristics}

Table 1 describes specific characteristics of the 23 studies included in this synthesis (authors and reference, country of origin, aim, design, data collection, analysis method, psychological construct(s) measured, measurement instruments, participant information, and the study's findings). The included studies reported outcomes for a total of 918 participants, with at least $70 \%$ being the mother of the child (two studies did not provide gender characteristics). Six studies were based on an experimental design with random assignment of participants to a control or treatment group, with the remaining studies using either a quasi-experimental pre-post design $(n=10)$, or mixed-methods design $(n=7)$. Studies included in the review were conducted in a total of 13 countries: Australia $(n=3)$, Canada $(n=1)$, China $(n=2)$, Greece $(n=1)$, India $(n=1)$, Iran $(n=3)$, Japan $(n=1)$, Jordan $(n=1)$, South Korean $(n=1)$, Spain $(n=1)$, Turkey $(n=1)$, United Kingdom $(n=1)$, and the United States $(n=6)$. 
Table 1. Characteristics of studies included in the synthesis.

\begin{tabular}{|c|c|c|c|c|}
\hline Reference, Year, Country & Aim & $\begin{array}{c}\text { Design/Data Collection/Analysis Psychological Construct } \\
\text { Measured: Instrument }\end{array}$ & Participants & Findings \\
\hline $\begin{array}{l}\text { Bitsika and Sharpley [46], } \\
\text { 2000, Australia }\end{array}$ & $\begin{array}{l}\text { To explore effect of parent support } \\
\text { program providing specific stress } \\
\text { management strategies }\end{array}$ & $\begin{array}{l}\text { Mixed design, within-subject/questionnaires, written } \\
\text { feedback/MANOVA content analysis } \\
\text { Anxiety: Self-Rating Anxiety Scale (SAS; Zung [47]) } \\
\text { Depression: Self-rating Depression Scale (SDS; Zung [48]) }\end{array}$ & $n=11$ mothers & $\begin{array}{l}\text { No significant change in stress, anxiety, } \\
\text { or depression but measure of group } \\
\text { cohesion increased; parent's written } \\
\text { feedback supported benefits gained from } \\
\text { learning specific stress management } \\
\text { technique (biofeedback) }\end{array}$ \\
\hline $\begin{array}{l}\text { Blackledge and Hayes [49], } \\
\text { 2006, United States }\end{array}$ & $\begin{array}{l}\text { To determine effectiveness of } \\
\text { Acceptance and Commitment } \\
\text { Therapy (ACT) on parent carers' } \\
\text { coping and mental health }\end{array}$ & $\begin{array}{l}\text { Within-subject, repeated } \\
\text { measures/questionnaires/Non-parametric Wilcoxon } \\
\text { signed-ranked test } \\
\text { Acceptance: Acceptance and Action Questionnaire } \\
\text { (AAQ; Hayes, Strosahl, et al. [50]) } \\
\text { Cognitions and automatic thoughts: Automatic Thoughts } \\
\text { Questionnaire (ATQ; Hollon and Kendall [51]) } \\
\text { Depression: Beck Depression Inventory II (BDI-II; Beck, Steer, } \\
\text { and Brown [52]) } \\
\text { General health: General Health Questionnaire-12 item } \\
\text { (GHQ-12; Goldberg [53]) } \\
\text { Locus of control: Parental Locus of Control Scale } \\
\text { (PLOS; Campis, Lyman, Prentice-Dunn [54]) } \\
\text { Psychological distress: Global Severity Index (GSI) from the } \\
\text { Brief Symptom Inventory (BSI; Derogatis and } \\
\text { Melisaratos [55]) }\end{array}$ & $n=20$ (15 mothers; 5 fathers) & $\begin{array}{l}\text { Improved psychological outcomes } \\
\text { retained at three month follow-up, } \\
\text { general distress and depression } \\
\text { decreased significantly, was most } \\
\text { pronounced among participants in } \\
\text { clinical range of depression }\end{array}$ \\
\hline $\begin{array}{l}\text { Clifford and Minnes [56], } \\
\text { 2013, Canada }\end{array}$ & $\begin{array}{l}\text { To investigate changes in parent } \\
\text { well-being following involvement in } \\
\text { on-line support group }\end{array}$ & $\begin{array}{l}\text { uasi-experimental between-group/questionnaires/MANOVA } \\
\text { Anxiety: State-Trait Anxiety Inventory (STAI; Spielberger [57]) } \\
\text { Depression: State-Trait Depression Scales } \\
\text { (STADS; Spielberger et al. [58]) } \\
\text { Family functioning: Kansas Inventory of Parental Perceptions } \\
\text { (KIPP; Behr et al. [59]) } \\
\text { Perceived stress: Family Stress and Coping Interview } \\
\text { (FSCI; Nachshen et al. [60]) }\end{array}$ & $\begin{array}{l}n=45 \text { ( } 43 \text { mothers, } 2 \text { fathers) } \\
\text { treatment group: } n=20 \\
\text { control group: } n=25\end{array}$ & $\begin{array}{l}\text { No significant differences on parenting } \\
\text { stress, anxiety, or depression }\end{array}$ \\
\hline $\begin{array}{l}\text { Erguner-Tekinalp and } \\
\text { Akkok [61], 2004, Turkey }\end{array}$ & $\begin{array}{l}\text { To explore effectiveness of coping } \\
\text { skills training on stress, coping skills, } \\
\text { and hopelessness among mothers }\end{array}$ & $\begin{array}{l}\text { Mixed method, between-group/questionnaires, } \\
\text { interviews/Mann Whitney U Test, content analysis } \\
\text { Coping: Coping Strategy Indicator (CSI; Amirkhan [62]) } \\
\text { Hopelessness: Beck Hopelessness Scale (BHS; Beck, Lester, } \\
\text { and Trexler [63]) } \\
\text { Parenting stress: Questionnaire on Resources and Stress } \\
\text { (QRS; Holroyd [64]) }\end{array}$ & $\begin{array}{l}n=20 \text { mothers; } \\
\text { Treatment group: } n=10, \\
\text { control group: } n=10\end{array}$ & $\begin{array}{l}\text { No significant difference on stress level, } \\
\text { but mothers in treatment group reported } \\
\text { an increase in use of social support as a } \\
\text { coping strategy, and felt a lower sense of } \\
\text { hopelessness }\end{array}$ \\
\hline $\begin{array}{l}\text { Farmer and Reupert [65], } \\
\text { 2013, Australia }\end{array}$ & $\begin{array}{l}\text { To decrease parent carers' anxiety } \\
\text { and increase confidence by } \\
\text { improving knowledge of ASD }\end{array}$ & $\begin{array}{l}\text { Mixed method, within-subjects/questionnaires/paired } t \text {-test, } \\
\text { inductive thematic analysis, } \\
\text { Self-efficacy and parenting anxiety: Understanding Autism } \\
\text { and Understanding my Child with Autism } \\
\text { (Farmer and Reupert [65]) }\end{array}$ & $\begin{array}{l}n=98 \text { ( } 63 \text { mothers, } 23 \text { fathers); } \\
86 \text { participants were } \\
\text { parents of child, } \\
12 \text { were other famly members }\end{array}$ & $\begin{array}{l}\text { Parental knowledge of ASD, and } \\
\text { confidence significantly increased, along } \\
\text { with a significant decrease in anxiety }\end{array}$ \\
\hline
\end{tabular}


Table 1. Cont.

\begin{tabular}{|c|c|c|c|c|}
\hline Reference, Year, Country & Aim & $\begin{array}{c}\text { Design/Data Collection/Analysis Psychological Construct } \\
\text { Measured: Instrument }\end{array}$ & Participants & Findings \\
\hline $\begin{array}{l}\text { Feinberg et al. [66], 2013, } \\
\text { United States }\end{array}$ & $\begin{array}{l}\text { To explore if maternal stress and } \\
\text { depressive symptoms would be } \\
\text { reduced as the result of strengthening } \\
\text { problem-solving skills }\end{array}$ & $\begin{array}{l}\text { Experimental design/questionnaires, interviews/comparison } \\
\text { of mean scores, } \\
\text { Chronbach alpha Coping: Brief Coping Orientation to } \\
\text { Problems (BCOP; Carver [67]) } \\
\text { Depression: Quick Inventory of Depressive Symptomatology } \\
\text { (QIDS; Rush et al. [68]) } \\
\text { Parenting stress: Parenting Stress Index-Short Form } \\
\text { (PSI-SF; Abidin [69]) }\end{array}$ & $\begin{array}{l}n=110 \text { mothers; } \\
\text { Treatment group: } n=59, \\
\text { Control group: } n=61\end{array}$ & $\begin{array}{l}\text { At three-month follow-up, mothers in the } \\
\text { problem-solving education group } \\
\text { reported higher use of social coping } \\
\text { (both instrumental and emotional), } \\
\text { and were significantly less likely than } \\
\text { mothers in the control group to report } \\
\text { clinically significant stress and } \\
\text { depressive symptoms. No change in } \\
\text { using problem-focused or avoidance } \\
\text { coping skills }\end{array}$ \\
\hline $\begin{array}{l}\text { Ferraioli and Harris [70], } \\
\text { 2013, United States }\end{array}$ & $\begin{array}{l}\text { To evaluate benefits of } \\
\text { mindfulness-based training approach } \\
\text { compared to skills-based parentental } \\
\text { training program on parental stress }\end{array}$ & $\begin{array}{l}\text { Experimental design, between-group, } \\
\text { within-subjects/questionnaires/independent } t \text {-tests, paired } \\
\text { samples } t \text {-test } \\
\text { General health: General Health Questionnaire-28 item } \\
\text { (GHQ-28; Goldberg and Williams [53]) } \\
\text { Mindfulness: Mindful Attention Awareness Scale } \\
\text { (MAAS; Brown and Ryan [71]) } \\
\text { Parenting stress: Parenting Stress Index-Short Form } \\
\text { (PSI-SF; Abidin [69]) }\end{array}$ & $\begin{array}{l}n=15 \text { (10 mothers, } 5 \text { fathers) } \\
\text { Mindfulness-based group: } n=6 \text {, } \\
\text { Skills-based group: } n=9\end{array}$ & $\begin{array}{l}\text { Parents in mindfulness-based group had } \\
\text { significant improvement over } \\
\text { skills-based group on measures of } \\
\text { parental stress and general health at } \\
\text { conclusion of training, and improved } \\
\text { general health at 3-month follow-up }\end{array}$ \\
\hline $\begin{array}{l}\text { Giarelli et al. [72], 2005, } \\
\text { United States }\end{array}$ & $\begin{array}{l}\text { To refine a parent-focused nursing } \\
\text { intervention and examine effects of } \\
\text { post-diagnosis nursing intervention } \\
\text { on parental psychological distress } \\
\text { and use of services }\end{array}$ & $\begin{array}{l}\text { Mixed methods, pretest-posttest experimental design, } \\
\text { observations and survey/questionnaires, } \\
\text { interview/non-parametric Wilcoxon test, content analysis } \\
\text { Perceived stress: Perceived Stress Scale } \\
\text { (PSS; Cohen and Williamson [73]) } \\
\text { Psychological distress: Impact of Events Scale } \\
\text { (IES; Zilberg et al. [74]) }\end{array}$ & $\begin{array}{l}n=31(16 \text { mothers, } 15 \text { fathers }) \\
\text { treatment group: } n=18 \\
\quad \text { control: } n=13\end{array}$ & $\begin{array}{l}\text { No significant differences on measures of } \\
\text { parental distress but parents in } \\
\text { intervention group reported being less } \\
\text { upset due to unexpected event of child's } \\
\text { diagnosis, the intervention group } \\
\text { increased their use of services when } \\
\text { compared to treatment-as-usual group } \\
\text { (control group). }\end{array}$ \\
\hline $\begin{array}{l}\text { Gika et al. [75], 2012, } \\
\text { Greece }\end{array}$ & $\begin{array}{l}\text { To examine impact of progressive } \\
\text { muscle/breathing relaxation } \\
\text { intervention on reduction of parental } \\
\text { and perceived stress }\end{array}$ & $\begin{array}{l}\text { Within-subjects, repeated } \\
\text { measures/questionnaires/non-parametric Wilcoxon } \\
\text { signed-rank test for dependent samples } \\
\text { Parenting stress: Parenting Stress Index-Short Form } \\
\text { (PSI-SF; Abidin [69]) } \\
\text { Perceived stress: Perceived Stress Scale-14 items } \\
\text { (PSS-14; Cohen, Kamarck and Mermelstein [76]) }\end{array}$ & $n=11 \mathrm{mothers}$ & $\begin{array}{l}\text { Both parental stress and perceived stress } \\
\text { was significantly reduced following } \\
\text { intervention }\end{array}$ \\
\hline $\begin{array}{l}\text { Izadi-Mazidi et al. [77], } \\
\text { 2015, Iran }\end{array}$ & $\begin{array}{l}\text { To examine the effectiveness of a } \\
\text { cognitive-behavior group therapy } \\
\text { intervention on parenting stress }\end{array}$ & $\begin{array}{l}\text { Within-subjects, repeated } \\
\text { measures/questionnaires/Independent samples } t \text {-test } \\
\text { Parenting stress: Parenting Stress Index-Short Form } \\
\text { (PSI-SF; Abidin [69]) }\end{array}$ & $n=16$ mothers & $\begin{array}{l}\text { Parenting stress and distress was } \\
\text { significantly reduced after intervention, } \\
\text { which included education about ASD, } \\
\text { discussions on cognitions and cognitive } \\
\text { errors, and the practice of relaxation }\end{array}$ \\
\hline
\end{tabular}


Table 1. Cont

\begin{tabular}{|c|c|c|c|c|}
\hline Reference, Year, Country & Aim & $\begin{array}{c}\text { Design/Data Collection/Analysis Psychological Construct } \\
\text { Measured: Instrument }\end{array}$ & Participants & Findings \\
\hline $\begin{array}{l}\text { Jamison et al. [78], 2017, } \\
\text { United States }\end{array}$ & $\begin{array}{l}\text { To evaluate a Family Peer Advocate } \\
\text { (FPA) model on improving parent } \\
\text { carers' utilization of services, } \\
\text { knowlege of ASD, and sense of } \\
\text { empowerment, and reducing } \\
\text { parenting stress }\end{array}$ & $\begin{array}{l}\text { Experimental design/questionnaires/Repeated } \\
\text { measures ANOVA } \\
\text { Caregiver stress or burden: Caregiver-Strain Questionnaire } \\
\text { (CSQ; Brannan et al. [79]) } \\
\text { Family functioning: Family Empowerment Scale } \\
\text { (FES; Karen et al. [80]) } \\
\text { Parenting stress: Parenting Stress Index-Short Form } \\
\text { (PSI-SF; Abidin [69]) } \\
\text { Social support: Social Support Survey (SSS; Sherbourne and } \\
\text { Steward [81]) }\end{array}$ & $\begin{array}{l}n=39 \text { racial/ethnic } \\
\text { minority parents; } \\
\text { Treatment group (i.e., FPA } \\
\text { assigned to family): } n=19 \\
\text { Control group: } n=20\end{array}$ & $\begin{array}{l}\text { Parenting stress in the treatment group } \\
\text { was significantly decreased as compared } \\
\text { to control group. No change was noted in } \\
\text { caregiver's sense of empowerment, or } \\
\text { use of services, although a lack of } \\
\text { available services in the community was } \\
\text { noted. Parent knowledge of ASD in both } \\
\text { groups increased }\end{array}$ \\
\hline Ji et al. [82], 2014, China & $\begin{array}{l}\text { To determine effectiveness of } \\
\text { multi-disciplinary parent education } \\
\text { program on improving health-related } \\
\text { quality of life (HRQOL) of } \\
\text { parent carers }\end{array}$ & $\begin{array}{l}\text { Quasi-experimental between-groups/questionnaires/ } \\
\text { independent } t \text {-test and paired samples } t \text {-test } \\
\text { Caregiver stress or burden: Caregiver Burden Index } \\
\text { (CBI; Novak and Guest [83]) } \\
\text { Coping: Simplified Coping Style Questionnaire } \\
\text { (SCSQ; Wang, Wang, and Ma [84]) } \\
\text { Family functioning: McMaster Family Assessment Device } \\
\text { (FAD; Epstein, Baldwin and Bishop [85]) } \\
\text { General health: Short Form Health Survey-36 items } \\
\text { (SF-36, Rand Health [86]) } \\
\text { Self-efficacy: General Self-Efficacy Scale } \\
\text { (GSE; Jerusalem and Schwarzer [87]) } \\
\text { Social support: Multidimensional Scale of Perceived Social } \\
\text { Support (MSPSS; Zimet, Powell, Farley, Werkman, } \\
\text { and Berkoff [88]) }\end{array}$ & $\begin{array}{c}n=42(38 \text { mothers, } 4 \text { fathers) } \\
\text { Treatment group: } n=22, \\
\text { Control group: } n=20\end{array}$ & $\begin{array}{l}\text { Significant improvement in mental } \\
\text { HRQOL, family functioning, self-efficacy, } \\
\text { and positive coping style were reflected } \\
\text { among participants in the } \\
\text { intervention group }\end{array}$ \\
\hline Joekar et al. [89], 016, Iran & $\begin{array}{l}\text { To determine effectiveness of } \\
\text { Acceptance and Commitment } \\
\text { Therapy (ACT) on Iranian parent } \\
\text { carers' coping and mental health }\end{array}$ & $\begin{array}{l}\text { Quasi-experimental between-group } \\
\text { design/questionnaires/MANCOVA } \\
\text { Acceptance: Acceptance and Action Questionnaire II } \\
\text { (AAQ-II; Bond et al. [90]) } \\
\text { General health: Short Form Health Survey-12 items } \\
\text { (SF-12, Rand Health [91]) }\end{array}$ & $\begin{array}{l}n=24 \text { mothers; } \\
\text { treatment group: } n=12, \\
\text { control group: } n=12\end{array}$ & $\begin{array}{l}\text { ACT was found to be effective in } \\
\text { decreasing symptoms of depression and } \\
\text { increasing psychological flexibility }\end{array}$ \\
\hline $\begin{array}{l}\text { Kim [92], 2016, South } \\
\text { Korea }\end{array}$ & $\begin{array}{l}\text { To examine the effects of the } \\
\text { Buddhist ontology focused } \\
\text { meditation programme on the } \\
\text { psychological health and well-being } \\
\text { of mothers of children with ASD }\end{array}$ & $\begin{array}{l}\text { Mixed methods, repeated measures, } \\
\text { within-subjects/questionnaires, interview/ANOVA, } \\
\text { content analysis } \\
\text { Affect: Positive and Negative Affect Schedule } \\
\text { (PANAS; Watson, Clark, and Tellegen [93]) } \\
\text { Depression: Depression, Anxiety, and Stress Scale } \\
\text { (DASS; Lovibond, and Lovibond [94]) } \\
\text { Anxiety: State-Trait Anxiety Inventory (STAI; Spielberger [57]) } \\
\text { Cognitions and automatic thoughts: Metacognitions } \\
\text { Questionnaire (MCQ; Cartwright-Hatton and Wells [95]) } \\
\text { Depression: Beck Depression Inventory II (BDI-II; Beck, Steer, } \\
\text { and Brown [52]) }\end{array}$ & $n=9$ mothers & $\begin{array}{l}\text { A statistically significant improvement } \\
\text { was found in psychological health } \\
\text { well-being and positive affect. } \\
\text { No changed was noted in anxiety or } \\
\text { negative affect. Participants reported that } \\
\text { the intervention helped them in relieving } \\
\text { feelings of guilt that they had done } \\
\text { wrong to accumulate bad karma and they } \\
\text { were more aware of trying to accept } \\
\text { people and things as they are. }\end{array}$ \\
\hline
\end{tabular}


Table 1. Cont.

\begin{tabular}{|c|c|c|c|c|}
\hline Reference, Year, Country & Aim & $\begin{array}{c}\text { Design/Data Collection/Analysis Psychological Construct } \\
\text { Measured: Instrument }\end{array}$ & Participants & Findings \\
\hline Lovell et al. [96], 2016, UK & $\begin{array}{l}\text { To examine the effectiveness of an } \\
\text { intervention on reducing } \\
\text { psychological distress in caregivers } \\
\text { through written emotional disclosure } \\
\text { regarding the benefits of caregiving }\end{array}$ & $\begin{array}{l}\text { Experimental design/questionnaires, written } \\
\text { essays/univariate ANOVA, chi square } \\
\text { Depression: Hospital Anxiety and Depression Scale } \\
\text { (HADS; Zigmond and Snaith [97]) } \\
\text { Perceived stress: Perceived Stress Scale } \\
\text { (PSS; Cohen and Williamson [73]) }\end{array}$ & $\begin{array}{c}n=33(28 \text { mothers, } 5 \text { fathers }) \\
\text { treatment group: } n=16 \\
\text { control group: } n=17\end{array}$ & $\begin{array}{l}\text { Anxiety scores for parent carers in the } \\
\text { benefit-finding condition were less likely } \\
\text { to be in the clinical range 3-months } \\
\text { post intervention }\end{array}$ \\
\hline $\begin{array}{l}\text { Nguyen et al. [98], 2016, } \\
\text { United States }\end{array}$ & $\begin{array}{l}\text { To determine if distress is reduced in } \\
\text { mothers of children recently } \\
\text { diagnosed with ASD following a } \\
\text { problem-solving skills } \\
\text { training program }\end{array}$ & $\begin{array}{l}\text { Within-group, repeated measures/questionnaires/Mixed } \\
\text { model analysis for repeated measures } \\
\text { Depression: Beck Depression Inventory-Revised (BDI-R; Beck, } \\
\text { Steer, and Brown [52]) } \\
\text { Mood: Profile of Mood States (POMS; McNair et al. [99]) } \\
\text { Psychological distress: Impact of Events Scale-Revised } \\
\text { (IES-R; Creamer, Bell, and Failla [100]) }\end{array}$ & $n=24$ mothers & $\begin{array}{l}\text { Mothers increased their effectiveness to } \\
\text { solve problems and experienced fewer } \\
\text { depressive symptoms over the course of } \\
\text { the study, less post-traumatic stress } \\
\text { symptoms and less disturbance of mood } \\
\text { was reported at 3-month follow-up but } \\
\text { problem-solving skills had decreased }\end{array}$ \\
\hline $\begin{array}{l}\text { Niimomi et al. [101], } \\
\text { 2016, Japan }\end{array}$ & $\begin{array}{l}\text { To determine the effectiveness of a } \\
\text { parenting support group program in } \\
\text { reducing parental stress and } \\
\text { improving quality of life }\end{array}$ & $\begin{array}{l}\text { Within-subjects design/questionnaires/Repeated-measure } \\
\text { multivariate analysis } \\
\text { Parenting stress: Parenting Stress Index-Short Form } \\
\text { (PSI-SF; Abidin [69]) } \\
\text { Quality of life: World Health Organization Quality of Life } \\
26 \text { item (WHOQOL-26; WHOQOL Group [102]) }\end{array}$ & $n=24$ mothers & $\begin{array}{l}\text { A significant increase in quality of life } \\
\text { was reported among participants at the } \\
\text { conclusion of the program and at } \\
\text { 3-months post follow-up. No change was } \\
\text { noted in reducing parental stress. }\end{array}$ \\
\hline $\begin{array}{l}\text { Patra et al. [103], } \\
\text { 2015, India }\end{array}$ & $\begin{array}{l}\text { To develop a psycho-educational } \\
\text { intervention for parents and to } \\
\text { determine it's impact on parent stress } \\
\text { and knowledge of ASD }\end{array}$ & $\begin{array}{l}\text { Mixed design, within-subjects/questionnaires, } \\
\text { interview/Wilcoxon-signed rank test, nominal group technique } \\
\text { Parenting stress: Family Interview for Stress and Coping } \\
\text { (FISC-MR; Girimaji, et al. [104]) }\end{array}$ & $\begin{array}{l}n=36 \text { married parents } \\
(18 \text { mothers, } 18 \text { fathers })\end{array}$ & $\begin{array}{l}\text { Parents' social stress and total stress } \\
\text { decreased by the conclusion } \\
\text { of intervention. }\end{array}$ \\
\hline $\begin{array}{l}\text { Ryan and Ahman [105], } \\
\text { 2016, Jordan }\end{array}$ & $\begin{array}{l}\text { To examine effectiveness of brief } \\
\text { mindfulness-based intervention on } \\
\text { parent carers perceived quality of life } \\
\text { (QoL) and positive stress reappraisal }\end{array}$ & $\begin{array}{l}\text { Quasi-experimental with non-equivalent control } \\
\text { group/questionnaires/Paired sample } t \text {-test, pre-post test } \\
\text { Coping: Cognitive Emotion Regulation Questionnaire } \\
\text { (CERQ; Garnefski, Kraaij, and Spinhoven [106]) } \\
\text { Mindfulness: Mindful Attention Awareness Scale } \\
\text { (MAAS; Brown and Ryan [71]) } \\
\text { Quality of life: World Health Organization Quality of } \\
\text { Life-Brief Version (WHOQOL-BREF; WHOQOL Group [107]) }\end{array}$ & $\begin{array}{c}n=110(73 \text { mothers; } 31 \text { fathers }) \\
\text { treatment group: } n=52 \\
\text { control group: } n=52\end{array}$ & $\begin{array}{l}\text { Physical health and environmental health } \\
\text { domains of QoL were not significant } \\
\text { between groups, but there was a } \\
\text { significant difference on psychological } \\
\text { health, social relationship health, and } \\
\text { overall QoL among participants in the } \\
\text { mindfulness intervention group }\end{array}$ \\
\hline
\end{tabular}


Table 1. Cont.

\begin{tabular}{|c|c|c|c|c|}
\hline Reference, Year, Country & Aim & $\begin{array}{c}\text { Design/Data Collection/Analysis Psychological Construct } \\
\text { Measured: Instrument }\end{array}$ & Participants & Findings \\
\hline $\begin{array}{l}\text { Ruiz-Robledillo et al. } \\
\text { [108], 2015, Spain }\end{array}$ & $\begin{array}{l}\text { To assess the effects of a mindfulness } \\
\text { intervention on the mood } \\
\text { disturbances and health complaints } \\
\text { among parent caregivers in } \\
\text { comparison to non-caregivers }\end{array}$ & $\begin{array}{l}\text { Quasi-experiemental, within-group, between-group } \\
\text { Anger; State-Trait Anger Expression Inventory-2 } \\
\text { (STAXI-2; Miguel-Tobal et al. [109]) } \\
\text { Anxiety: State-Trait Anxiety Inventory (STAI; Spielberger [57]) } \\
\text { Caregiver stress or burden: Zarit Burden Inventory } \\
\text { (ZBI; Zarit et al. [110]) } \\
\text { Depression: Beck Depression Inventory } \\
\text { (BDI; Beck and Steer [111]) } \\
\text { General health: General Health Questionnaire-28 item } \\
\text { (GHQ-28; Goldberg and Williams [53]) } \\
\text { Mood: Profile of Mood States (POMS; McNair et al. [99]) } \\
\text { Somatic symptoms: Somatic Symptoms Scale-Revised } \\
\text { (Spanish Version) (ESS-R; Sandin and Chorot [112]) }\end{array}$ & $\begin{array}{l}n=13 \text { (13 mothers; } 1 \text { father }) \\
\text { Caregiver group: } n=6 \\
\text { (5 mothers, } 1 \text { father }) \\
\text { Non-Caregiver group: } n=7 \\
\text { (7 mothers) }\end{array}$ & $\begin{array}{l}\text { A significant reduction in mood } \\
\text { disturbances and afternoon cortisol levels } \\
\text { occurred for all participants, but were } \\
\text { more pronounced in the caregiver group } \\
\text { of parents. All participants reported a } \\
\text { decrease in depressive and somatic } \\
\text { symptoms at the end of the program and } \\
\text { improved self-perceived general health. }\end{array}$ \\
\hline $\begin{array}{l}\text { Samadi et al. [113], } \\
\text { 2012, Iran }\end{array}$ & $\begin{array}{l}\text { To determine the effectiveness of a } \\
\text { short group-based support course } \\
\text { designed to increase parent's } \\
\text { knowledge of ASD and interventions } \\
\text { to promote child's development, } \\
\text { boost parent's confidence and sense } \\
\text { of empowerment, and encourage } \\
\text { parents to provide informal support } \\
\text { to each other }\end{array}$ & $\begin{array}{l}\text { Mixed design included pre-post, crossover design, } \\
\text { within-group differences, between-group differences, } \\
\text { questionnaires, interviews, paired } t \text {-tests, independent } t \text {-tests, } \\
\text { content analysis } \\
\text { Coping: Coping Styles Questionnaire (CSQ; Roger, Jervis and } \\
\text { Najarian [114]) } \\
\text { Family functioning: McMaster Family Assessment Device } \\
\text { (FAD; Epstein, Baldwin and Bishop [85]) } \\
\text { General health: General Health Questionnaire-28 item } \\
\text { (GHQ-28; Goldberg and Williams [53]) } \\
\text { Parenting stress: Parenting Stress Index-Short Form } \\
\text { (PSI-SF; Abidin [69]) }\end{array}$ & $n=37$ (24 mothers, 13 fathers) & $\begin{array}{l}\text { Intervention resulted in significant } \\
\text { improvements on parental ratings of } \\
\text { health, stress, and family functioning, } \\
\text { and a significant increase in use of } \\
\text { problem-focused coping strategies up to } \\
\text { 4-months post intervention }\end{array}$ \\
\hline
\end{tabular}


Table 1. Cont.

\begin{tabular}{|c|c|c|c|c|}
\hline Reference, Year, Country & Aim & $\begin{array}{c}\text { Design/Data Collection/Analysis Psychological Construct } \\
\text { Measured: Instrument }\end{array}$ & Participants & Findings \\
\hline $\begin{array}{l}\text { McConkey and Samadi } \\
\text { [115], 2013, IranNOTE: } \\
\text { This study is a 12-month } \\
\text { follow-up study to } \\
\text { Samadi et al. [113] and } \\
\text { thus was not counted as a } \\
\text { separate intervention } \\
\text { study in the overall } \\
\text { number of studies. }\end{array}$ & $\begin{array}{l}\text { To determine the extent of informal } \\
\text { support among Iranian parents who } \\
\text { had a child with ASD following a } \\
\text { group-based training course and if } \\
\text { indicators of parental well-being and } \\
\text { use of problem-focused and } \\
\text { emotional focused coping strategies } \\
\text { were maintained } 12 \text { months } \\
\text { post-intervention }\end{array}$ & $\begin{array}{l}\text { Mixed design included pre-post, crossover design, } \\
\text { within-group differences, between-group } \\
\text { differences/questionnaires, interviews/paired } t \text {-tests, } \\
\text { independent } t \text {-tests, thematic analysis } \\
\text { Coping: Coping Styles Questionnaire } \\
\text { (CSQ; Roger, Jervis and Najarian [114]) } \\
\text { Family functioning: McMaster Family Assessment Device } \\
\text { (FAD; Epstein, Baldwin and Bishop [85]) } \\
\text { Family health: General Health Questionnaire-28 item } \\
\text { (GHQ-28; Goldberg and Williams [53]) } \\
\text { Parenting stress: Parenting Stress Index-Short Form } \\
\text { (PSI-SF; Abidin [69]) }\end{array}$ & $n=28$ (17 mothers, 11 fathers $)$ & $\begin{array}{l}\text { Improved family functioning and better } \\
\text { health of parents was maintained up to } \\
12 \text { months post-intervention, particularly } \\
\text { among those parents who had } \\
\text { maintained contact with others in the } \\
\text { initial group-based intervention; } \\
\text { child-related stress levels returned to } \\
\text { baseline regardless if contact had been } \\
\text { maintained; problem-focused coping } \\
\text { strategies were not maintained } \\
12 \text { months-post intervention }\end{array}$ \\
\hline $\begin{array}{l}\text { Tongue et al. [116], 2006, } \\
\text { Australia }\end{array}$ & $\begin{array}{l}\text { To determine the impact of a parent } \\
\text { education and behavior management } \\
\text { intervention (PEBM), and a parent } \\
\text { education and counseling } \\
\text { intervention (PEC) on the mental } \\
\text { health and adjustment of parent } \\
\text { carers with preschool children with } \\
\text { autism }\end{array}$ & $\begin{array}{l}\text { Experimental design/questionnaires/Analysis of Covariance } \\
\text { Family functioning: McMaster Family Assessment Device } \\
\text { (FAD; Epstein, Baldwin and Bishop [85]) } \\
\text { Family health: General Health Questionnaire-28 item } \\
\text { (GHQ-28; Goldberg and Williams [53]) } \\
\text { Parenting stress: Parenting Stress Thermometer [116] } \\
\text { [visual analogue rating of general stress from } 0 \text { (none) to } 4 \\
\text { (very, very much)] }\end{array}$ & $\begin{array}{l}n=105 \text { (info on gender of } \\
\text { sample not reported) } \\
\text { PEBM group: } n=35 \\
\text { PEC group: } n=35 \\
\text { Control group: } n=35\end{array}$ & $\begin{array}{l}\text { Both the PEBM and PEC interventions } \\
\text { contained the same educational material, } \\
\text { however the PEBM intervention included } \\
\text { sessions that were skills based and action } \\
\text { oriented while the PEC intervention } \\
\text { emphasized nondirective, interactive } \\
\text { discussion and counseling. There were } \\
\text { no significant differences between the } \\
\text { outcomes of the PEBM and PEC } \\
\text { groups-both interventions reduced } \\
\text { overall symptoms of parental distress, } \\
\text { although the PEC intervention reduced } \\
\text { the depressive symptoms in a larger } \\
\text { percentage of parents. }\end{array}$ \\
\hline $\begin{array}{l}\text { Zhang et al. [117], } \\
\text { 2014, China }\end{array}$ & $\begin{array}{l}\text { To evaluate the effectiveness of a } \\
\text { group-based solution-focused brief } \\
\text { therapy on promoting } \\
\text { post-traumatic growth }\end{array}$ & $\begin{array}{l}\text { Experimental design/questionnaires/independent } \\
\text { samples } t \text {-test } \\
\text { Post-traumatic growth: Post-Traumatic Growth } \\
\text { Inventory-Chinese version } \\
\text { (PTGI-C; Tedeschi and Calhoun [118]) }\end{array}$ & $\begin{array}{c}n=45 \text { mothers; } \\
\text { Treatment groups }=20 \\
(2 \text { groups of } 10), \\
\text { Control group }=25\end{array}$ & $\begin{array}{l}\text { Post-traumatic growth was significantly } \\
\text { higher among mothers in the } \\
\text { intervention groups compared to control } \\
\text { group. The difference was maintained at } \\
\text { a 3-months follow-up. }\end{array}$ \\
\hline
\end{tabular}




\subsection{Data Extraction}

In order to ensure that none of the key aspects of the studies were overlooked, a grid was developed (see Table 2) that allowed for the recording of key concepts from each study based on the nature of the intervention, results of the study, perspectives reported by the participants, and the conclusions and observations of the investigators. Columns were constructed with the top row identifying the study's citation number, while the rows of the grid listed the key concepts drawn from each study. The concepts were then integrated across studies through a series of discussions among the co-authors regarding the similarities and differences of the individual concepts within the context of the various studies, producing a reduced number of transformed synthetic concepts which were then grouped together into a structure of higher order themes. These overarching, higher order themes identified a synthesized interpretation representative of the whole body of evidence.

\subsection{Synthesis}

Three major themes were identified as being central to improving the mental health of parents who have a child affected by ASD-access to social support with similar parent carers, receiving professional stress management and problem-solving training; and the provision of accurate information regarding ASD. Table 2 lists the major themes and their subthemes resulting from the integrated synthesis of evidence reported in the individual studies. The top row indicates the citation for the study.

\subsubsection{Social Support}

The role of social support was found to be an important factor in twelve studies that either specifically examined the role of social support in improving the psychological wellbeing of parents or included a group treatment condition in which parents were able to engage in discussions with other parent carers. The theme of social support encompassed the roles of informal networks, reduced isolation, and validation by similar parent carers.

\section{Informal Networks}

Seven studies $[46,56,65,101,103,113,116]$ involved an intervention examining the role of social support and found parent carers involved in a parenting social group also reported decreased anxiety [65] and social stress [103,113], increased group cohesion [46], and improved health and family functioning [113] and quality of life [101]. The benefits of a parenting support group on measures of parental well-being was found to be maintained up to twelve months post intervention [115]. An asynchronous on-line support group appeared to not to be effective in reducing parenting stress, anxiety, or depression [56], suggesting the beneficial effect of social support may involve direct, real-time communication methods (e.g., face-to-face, telephone calls).

\section{Reduced Isolation}

Five studies found social support related to improvements in parents' psychological wellbeing was attributed to the increased awareness by parents that they were not alone in the challenges they faced parenting a child affected by ASD. Parents who reported feeling less socially isolated because of having access to other parents who were also engaged in the intervention reported a decrease in anxiety and stress $[46,61,65,113,116]$.

\section{Validation by Peers}

Feedback from parents in eight studies $[46,61,65,77,92,113,116,117]$ suggests that the validation they received from the other parents as an important factor to improvements of their wellbeing. These studies found social support was associated with increased group cohesion [46], confidence [65], and post-traumatic growth [117], as well as less hopelessness [61], stress [77,78,113], anxiety [61], 
depression [116]. The normalizing effect due to receiving support and empathy from other group members was reported by parents as a factor in facilitating their ability to cope and adjust their perspective to the parenting challenges they faced. Parents were able to recognize that their experience was not all negative but rather a different "normal".

\subsubsection{Professional Training in Skill Development}

Fourteen studies included professionally-led workshops based on cognitive-behavioral approaches that provided parents with various stress management strategies (nine studies), and problem-solving skill training (six studies).

\section{Stress Management Strategies}

Nine studies $[46,49,61,70,75,92,96,105,108]$ involved interventions that provided training in various stress management strategies. Biofeedback was found to increased group cohesion and reportedly provided parents with a helpful strategy for dealing with their day to day stress [46], while mindfulness was associated with decreased mood disturbances [111], and increased psychological [92,105] and general health [70], as well as overall quality of life [105]. Progressive muscle relaxation was associated with decreased stress [75], and expressive writing, particularly writing related to describing the benefits of being a parent carer, was associated with decreased anxiety [96]. Interventions incorporating Acceptance and Commitment therapy, found the approach associated with decreased depression and distress, and increased psychological flexibility $[49,89]$.

\section{Problem-Solving Skills}

Five studies $[61,65,66,98,117]$ involved parents receiving training in problem-solving or coping strategies. These interventions were reported to be particularly useful to parents when the training was structured and focused on providing practical knowledge and skills for dealing with their child's behavioral problems and daily care. Skill training in problem-solving strategies appeared to be associated with parents' increased use of social support [61,66], increased confidence in addressing problems [65], promoted post-traumatic growth [117], and decreased parent carers' levels of anxiety [65], stress [66], and depression [98].

\subsubsection{Gaining Knowledge Regarding ASD}

Eight studies $[61,65,72,77,78,103,113,116]$ examined the influence of providing parent carers with information about ASD and the types of resources and services that were available to them as a strategy for reducing parental stress and anxiety.

\section{Understanding Autism Spectrum Disorder}

Five studies $[65,77,78,113,116]$ involved an intervention in which parents were provided with information regarding ASD and its related cognitive, emotional, and behavioral features. As parents' knowledge of ASD increased, stress, anxiety and distress was reduced $[65,77,78,116]$, confidence and ratings of health increased [65,113], and the use of problem-solving skills increased [113].

\section{Resources and Services}

Four studies $[65,72,78,103]$ involved interventions providing parents information about available resources and services from healthcare professionals as a strategy to address parental stress and anxiety. Parents who were provided with information regarding advocacy services, such as available educational, developmental, and behavioral treatment programs, and had assistance in identifying ways to fill service gaps reported a decrease in anxiety [65,78], stress [103], and were less distressed upon receiving their child's diagnosis [72]. 
Table 2. Breakdown of studies into themes.

\begin{tabular}{|c|c|c|c|c|c|c|c|c|c|c|c|c|c|c|c|c|c|c|c|c|c|c|c|}
\hline Theme and Subthemes & 47 & 50 & 57 & 62 & 66 & 67 & 71 & 74 & 77 & 79 & 80 & 84 & 91 & 94 & 98 & 100 & 104 & 106 & 108 & 111 & $116 / 118$ & 119 & 120 \\
\hline \multicolumn{24}{|l|}{ Importance of social support } \\
\hline Informal networks & + & & + & & + & & & & & & & & & & & & + & + & & & + & + & \\
\hline Reduced isolation & + & & + & + & + & & & & & & & & & & & & & & & & + & + & \\
\hline Validation by peers & + & & + & + & + & & & & & + & & & & + & & & & & & & + & & + \\
\hline \multicolumn{24}{|l|}{ Skill development } \\
\hline Acceptance/Stress management & + & + & & + & & & + & & + & & & & + & + & + & & & & + & + & & & \\
\hline Problem-solving & + & + & & + & + & + & + & & & & & + & & & & + & & & & & & & + \\
\hline \multicolumn{24}{|l|}{ Gaining knowledge } \\
\hline ASD & & & & & + & & & + & & + & & & & & & & & + & & & + & & \\
\hline Resources, services & & & & + & & & & + & & & + & & & & & & & + & & & & & \\
\hline
\end{tabular}




\section{Discussion}

This systematic review applied a critical interpretive synthesis analysis that included both quantitative and qualitative studies in order to triangulate emerging evidence, and to formulate needed practice guidelines for health and allied-health professionals who provide mental health support to parent carers of a child affected by ASD. Three broad themes emerged from this synthesis of primary studies-the importance of social support by other parent carers, the effectiveness of training parents in stress management strategies and developing problem-solving skills, and the importance of providing parent carers with relevant and accurate information about ASD and available resources and support services. These themes can be effectively incorporated into strategies practitioners and their parent carer partners can utilize for improving not only the psychological well-being of parent carers, but also to influence the wellbeing of the child affected by ASD and other family members.

Perhaps one the most effective factors identified in the synthesis found to influence the well-being of parent carers was being engaged with other parent carers, such as through a parenting social support group. Networking with other parent carers allowed for parents to realize they were not alone in the challenges they faced [56] and provided parents with an important validation of their own value and experiences $[56,61,65]$. They could share stories with others who may have had similar experiences and discuss life's difficulties $[46,61,65,66]$, exchange ideas and information about resources $[46,61]$, and learn from other parents how they respond and cope with their child's behaviors, as well as the criticism often received from others regarding their child's behavior [46,61]. Through the support of other similar parent carers, parents are able to normalize their experience and become more aware of their child's needs rather than focusing on a comparison of their child to other children $[65,77,92,113]$. Receiving validation from others helped increase parent carers' confidence to cope with daily challenges and be more accepting of their child's behaviors [46,92]. Parents could also notice the positive changes in each other and provide continued support in the growth and well-being of the other parent carers [56].

Participating in professionally-led workshops in which parent carers were introduced to the practice of various stress management strategies (e.g., biofeedback, mindfulness), acceptance, and learned problem-solving skill strategies, had a positive effect on parents' mental health and psychological well-being $[46,49,61,70,75,89,92,96,105,108]$. Helping parents experience the practice and value of "being present", rather than ruminating over unresolved events in the past, or activating thoughts about anticipated future events, enhances the parents' ability to stay focused on the present situation in a non-judgmental way and promotes an awareness of choices the parent carer may have in response to a situation. Coupled with training in problem-solving strategies, providing training in stress management strategies has the potential to improve parents carers' wellbeing by increasing their confidence in effectively managing daily challenges, and their ability to be more accepting of others, including their child as well as themselves.

Workshops providing training in stress management strategies and problem-solving skills also functioned as social support groups, providing participants with opportunities to develop and practice new problem-solving strategies to the challenges they routinely faced while also learning from other group members about their experiences and strategies. Overall, stress management strategies helped parent carers learn a beneficial strategy for managing stress they were able to practice in their own home and utilize whenever they found themselves facing a difficult situation. Additionally, workshops provided parent carers with an opportunity for respite from their daily caregiving responsibilities.

Parent carers reported that receiving factual and accurate information regarding ASD helped decrease their feelings of stress and anxiety, often expressing relief after receiving information that helped explain their child's behaviors. Providing parents with a better understanding of how their child processed sensory information, and the relationship of that experience to the child's engagement in certain behaviors, was reported as making a difference in how parents related and reacted to their child, promoting better acceptance and understanding of the child behavior. When provided with an explanation that related their child's diagnosis to the child's behavior, parents' confidence in seeking appropriate services increased. With accurate and relevant knowledge about the nature of 
ASD, parents described being able to develop more effective strategies and tools that helped their child better cope with the sensory stimulation they experienced in their environment. Learning about the sensory aspects of autism and strategies that would help to support their child was reported as being highly valued by parent carers.

When developing a treatment plan to address the mental health needs of a parent carers with a child affected by ASD, including any, or all, of the above strategies, should be considered. Improved mental health and psychological well-being was reported by parent carers as a result of each of these strategies, often with strategies, such as receiving social support from similar parent carers, being imbedded into other strategies, such as group workshops on stress management and problem-solving. These strategies can form the framework for the development of strategies that could be delivered to parent carers of a child affected with ASD and perhaps expanded to address parent carers of children with other forms of neurodevelopmental disorders and chronic illness.

\subsection{Strengths and Limitations of the Review}

This review used a rigorous review method for a critical integrative synthesis involving a comprehensive search of published studies examining the effectiveness of an intervention designed to improve the mental health and psychological wellbeing of parents of a child affected with ASD and that met pre-determined inclusion criteria. Key factors were identified from a diverse body of literature that included both quantitative effectiveness studies as well as qualitative studies that provided the participants' perspectives regarding the benefits and limitations of the interventions, as well as their own beliefs, values, and feelings about their experience with the intervention. Although each study occurred within a different context, we were able to integrate and synthesize findings across primary studies in order to identify higher level constructs that could provide a more comprehensive understanding of the factors involved in effective interventions for addressing the psychological well-being of parents with a child affected by ASD.

It is acknowledged that the results of this study should be considered a beginning step in building a body of knowledge that would prove useful to health and professional mental health practitioners who provide support services to parent carers and their child affected by ASD. In spite of conducting a comprehensive search of the evidence, a synthesis of all forms of evidence is the desired standard, such as unpublished studies, dissertations, single-case studies, and conference presentations, to name a few. Such an undertaking, however, was not feasible given the time and number of personnel involved, thus it was necessary to limit the sampling of evidence to those that met our pre-determined criteria with the hopes that others will continue to expand upon the evidence we have provided.

It is also possible that others might have reviewed the studies included in the present synthesis and identified alternative overarching constructs. The purpose of a critical integrative synthesis is to generate theory [42], and we hope our findings will prove useful in the development of a theory that provides strong explanatory power regarding those factors that must be present in an effective, and efficient, strategy for enhancing the psychological well-being of parents who are experiencing significant distress due to the challenges of parenting a child affected by ASD. For example, there is a paucity of research examining the mental health experience of those parents who live in rural areas and have less access to resources, or are of a lower socioeconomic status, or belong to a minority ethnic population. Additional qualitative research is needed to provide the valuable perspective and insights of parent carers, which should lead to developing randomized controlled studies in order to compare intervention strategies and determine what works for whom and under what circumstances.

\subsection{Implications of the Review and Practice Guidelines}

This synthesis identifies several strategies that could be implemented by practitioners immediately in order to improve the mental health of parent carers of a child affected by ASD. First, incorporating an opportunity for parents to interact with other similar parents appears to be a very beneficial strategy for enhancing parents' psychological wellbeing. Facilities providing behavioral services to children 
with ASD should consider integrating support services for parents, such as forming parental support groups, in addition to the behavioral services being delivered to the child affected by ASD. Having a practitioner who is knowledgeable about ASD, and familiar with the issues and challenges parent carers face caring for a child affected by ASD, lead or participate in the support group would be beneficial so that parents could ask questions and obtain relevant and accurate information about ASD. Many parents may lack available child care and having a parent support group available at the facility where their child is receiving behavioral services may be the parent's only opportunity for beneficial social support and access to information. Parents should be encouraged to form relationships with the other parents so that the provision of peer support could potentially continue as the child ages and parents face new challenges. Practitioners could develop and lead parent support groups that include activities and training in various skills to enhance stress management, coping, and problem-solving practices. Incorporating parent support services formerly into the services being provided to the child at the treatment facility could be an important factor for facilitating the success of the child's treatment. Secondly, practitioners could serve as advocates for parents and help them find appropriate services and resources to ensure there are no gaps in services for the child. Health and mental health professional practitioners should discuss with parent carers the different aspects of their quality of life and ensure the parent is referred for appropriate services. Thirdly, health and mental health practitioners should provide parents with written information regarding the nature of ASD that could be shared with other family members, as well as guidelines for problem-solving, strategies for stress management, and available resources and services that might be beneficial for either the child or the parent. Treatment facilities that deliver behavioral interventions for children with ASD might consider integrating these described strategies into a package of mental health support services that parent carers could receive, ensuring that the wellbeing of both the child and parent carers are being effectively addressed.

\section{Conclusions}

Parents providing ongoing care to a child affected by ASD are found to be at higher risk for adverse mental health outcomes such as chronic stress, depression, and anxiety. Practice guidelines for the use by practitioners and parent carers of children with ASD should address the need for engaging with other similar parent carers, and enhance their generic problem-solving abilities, self-perspective taking, and sense of meaning as carers. Practitioners who address the state of the parent's mental health and psychological well-being will enhance the health-related quality of life of the parent carers, their families and their child with ASD. The implementation of the guidelines proposed in this integrative synthesis can potentially lead to greater/better cooperation with child care services, as well as an improved quality of life for parent carers and their child.

Acknowledgments: No funding was received by any co-author in support of this research project or work.

Author Contributions: Denise Catalano conducted the search for literature, reviewed articles to determine eligibility and assessed quality of studies for inclusion in this systematic review, constructed the tables and thematic grid, contributed to the synthesis of concepts and development of themes, and contributed to the writing of this paper. Linda Holloway reviewed articles to determine eligibility and assessed quality of studies for inclusion in the review, contributed to the synthesis of concepts and development of themes, and reviewed and edited drafts of this paper. Elias Mpofu initiated the project, contributed to the synthesis of concepts and development of themes, and contributed to the writing and editing of this paper.

Conflicts of Interest: The authors declare no conflict of interest.

\section{References}

1. Brobst, J.B.; Clopton, J.R.; Hendrick, S.S. Parenting children with autism spectrum disorders: The couple's relationship. Focus Autism Other Dev. Disabil. 2009, 24, 38-49. [CrossRef] 
2. Hoffman, C.D.; Sweeney, D.P.; Hodge, D.; Lopez-Wagner, M.D.; Looney, L. Parenting stress and closeness: Mothers of typically developing children and mothers of children with autism. Focus Autism Other Dev. Disabil. 2009, 24, 178-187. [CrossRef]

3. Lee, G.K.; Lopata, C.; Volker, M.A.; Thomeer, M.L.; Nida, R.E.; Toomey, J.A.; Chow, S.Y.; Smerbeck, A.M. Health-Related quality of life of parents of children with high-functioning autism spectrum disorders. Focus Autism Other Dev. Disabil. 2009, 24, 227-239. [CrossRef]

4. Abbeduto, L.; Seltzer, M.M.; Shattuck, P.; Krauss, M.W.; Orsmond, G.; Murphy, M.M. Psychological well-being and coping in mothers of youths with autism, Down syndrome, or fragile X syndrome. Am. J. Ment. Retard. 2004, 109, 237-254. [CrossRef]

5. Pisula, E. A comparative study of stress profiles in mothers of children with autism and those of children with Down's syndrome. J. Appl. Res. Intellect. Disabil. 2007, 20, 274-278. [CrossRef]

6. Cochrane, J.J.; Goering, P.N.; Rogers, J.M. The mental health of information caregivers in Ontario: An epidemiological survey. Am. J. Public Health 1997, 87, 2002-2007. [CrossRef] [PubMed]

7. Pinquart, M.; Sörensen, S. Differences between caregivers and noncaregivers in psychological health and physical health: A meta-analysis. Psychol. Aging 2003, 18, 250-267. [CrossRef] [PubMed]

8. Davis, N.O.; Carter, A.S. Parenting stress in mothers and fathers of toddlers with autism spectrum disorders: Association with child characteristics. J. Autism Dev. Disord. 2008, 38, 1278-1291. [CrossRef] [PubMed]

9. Hayes, S.A.; Watson, S.L. The impact of parenting stress: A meta-analysis of studies comparing the experience of parenting stress in parents of children with and without autism spectrum disorder. J. Autism Dev. Disord. 2013, 43, 629-642. [CrossRef] [PubMed]

10. Lovell, B.; Moss, M.; Wetherell, M. The psychosocial, endocrine and immune consequences of caring for a child with autism or DAH. Psychoneuroendocrinology 2012, 17, 534-542. [CrossRef] [PubMed]

11. Ruiz-Robledillo, N.; Moya-Albiol, L. Self-Reported health and cortisol awakening response in parents of people with Asperger syndrome: The role of trait anger and anxiety, coping and burden. Psychol. Health 2013, 28, 1246-1264. [CrossRef] [PubMed]

12. American Psychiatric Association. Neurodevelopmental disorders: Autism spectrum disorder. In Diagnostic and Statistical Manual of Mental Disorders, 5th ed.; American Psychological Association: Arlington, VA, USA, 2013.

13. World Health Organization. Available online: http://www.who.int/mediacentre/factsheets/autismspectrum-disorders/en/ (accessed on 19 January 2018).

14. Statistia. Available online: https:/ / www.statista.com/statistics/676354/autism-rate-among-children-selectcountries-worldwide/ (accessed on 19 January 2018).

15. Centers for Disease Control and Prevention. Available online: https://www.cdc.gov/ncbddd/autism/ addm.html (accessed on 19 January 2018).

16. Gray, D.E. Gender and coping: The parents of children with high functioning autism. Soc. Sci. Med. 2003, 56, 631-642. [CrossRef]

17. Exhaustion, Anger of Caregiving Get a Name. Available online: http:/ / www.cnn.com/2007/HEALTH/ conditions/08/13/caregiver.syndrome/index.html (accessed on 20 September 2017).

18. Killian, D. Helping ill it hurts? A multimethod study of compassion fatigue, burnout, and self-care in clinicians working with trauma survivors. Traumatology 2008, 14, 32-44. [CrossRef]

19. Lawton, M.P.; Moss, M.; Kleban, M.H.; Glicksman, A.; Rovine, M. A two-factor model of caregiving appraisal and psychological well-being. J. Gerontol. 1991, 46, 181-189. [CrossRef]

20. Quintero, N.; McIntyre, L.L. Sibling adjustment and maternal well-being: An examination of families with and without a child with an autism spectrum disorder. Focus Autism Other Dev. Disabil. 2010, 25, 37-46. [CrossRef] [PubMed]

21. Baker, J.K.; Seltzer, M.M.; Greenberg, J.S. Longitudinal effects of adaptability on behavior problems and maternal depression I families of adolescents with autism. J. Fam. Psychol. 2011, 25, 601-609. [CrossRef] [PubMed]

22. Bitsika, V.; Sharpley, C. An exploratory examination of the effects of support groups on the well-being of parents of children with autism-I: General counselling. J. Appl. Health Behav. Sci. 1999, 1, 16-22.

23. Eisenhower, A.S.; Baker, B.L.; Blacher, J. Preschool children with intellectual disability: Syndrome specificity, behavior problems, and maternal well-being. J. Intellect. Disabil. Res. 2005, 49, 657-671. [CrossRef] [PubMed] 
24. Estes, A.; Munson, J.; Dawson, G.; Koehler, E.; Zhou, X.; Abbott, R. Parenting stress and psychological functioning among mothers of preschool children with autism and developmental delay. Autism 2009, 13, 375-387. [CrossRef] [PubMed]

25. Hamlyn-Wright, S.; Draghi-Lorenz, R.; Ellis, J. Locus of control fails to mediate between stress and anxiety and depression in parents of children with a developmental disorder. Autism 2007, 11, 489-501. [CrossRef] [PubMed]

26. Mugno, D.; Ruta, L.; D-Arrigo, V.G.; Mazzone, L. Impairment of quality of life in parents of children and adolescents with pervasive developmental disorder. Health Qual. Life Outcomes 2007, 5. [CrossRef] [PubMed]

27. Weiss, M.J. Hardiness and social support as predictors of stress in mothers of typical children, children with autism, and children with mental retardation. Autism 2002, 6, 115-130. [CrossRef] [PubMed]

28. Smith, L.E.; Hong, J.; Seltzer, M.M.; Greenberg, J.S.; Almeida, D.M.; Bishop, S.L. Daily experiences among mothers of adolescents and adults with autism spectrum disorder. J. Autism Dev. Disord. 2010, 40, 167-178. [CrossRef] [PubMed]

29. Khanna, R.; Madhavan, S.S.; Smith, M.J.; Patrick, J.H.; Tworek, C.; Becker-Cottrill, B. Assessment of health-related quality of life among primary caregivers of children with autism spectrum disorders. J. Autism Dev. Disord. 2011, 41, 1214-1227. [CrossRef] [PubMed]

30. Allik, H.; Larsson, J.-O.; Smedje, H. Health-Related quality of life in parents of school-age children with Asperger syndrome or high-functioning autism. Health Qual. Life Outcomes 2006, 4. [CrossRef] [PubMed]

31. Fong, L.; Wilgosh, L.S.; Sobsey, D. The experience of parenting an adolescent with autism. Int. J. Disabil. Dev. Educ. 1993, 40, 105-113. [CrossRef]

32. Kuhaneck, H.M.; Madonna, S.; Novak, A.; Pearson, E. Effectiveness of interventions for children with Autism Spectrum Disorder and their parents: A systematic review of family outcomes. Am. J. Occup. Ther. 2015, 69, 1-12. [CrossRef] [PubMed]

33. Meltzer, L.J. Factors associated with depressive symptoms in parents of children with Autism Spectrum Disorders. Res. Autism Spectr. Disord. 2011, 5, 361-367. [CrossRef]

34. Serrata, C.A. Psychosocial aspects of parenting a child with autism. J. Appl. Rehabil. Couns. 2012, 43, $29-35$.

35. Campbell, J.M. Efficacy of behavioral intervention for reducing problematic behaviors in persons with autism: A quantitative synthesis of single-subject research. Res. Dev. Disabil. 2003, 24, 120-138. [CrossRef]

36. Leyfer, O.T.; Folstein, S.E.; Bacalman, S.; Davis, N.O.; Dinh, E.; Morgan, J.; Tager-Flusberg, H.; Lainhart, J.E. Comorbid psychiatric disorders in children with autism: Interview development and rates of disorders. J. Autism Dev. Disord. 2006, 36, 849-861. [CrossRef] [PubMed]

37. Baker, J.K.; Seltzer, M.M.; Greenberg, J.S. Behavior problems, maternal internalizing symptoms and family relations in families of adolescents and adults with fragile X syndrome. J. Intellect. Disabil. Res. 2012, 56, 984-995. [CrossRef] [PubMed]

38. Jellett, R.; Wood, C.E.; Giallo, R.; Seymour, M. Family functioning and behavior problems in children with Autism Spectrum Disorders: The mediating role of parent mental health. Clin. Psychol. 2015, 19, 39-48. [CrossRef]

39. Ooi, K.L.; Ong, Y.S.; Jacob, S.A.; Khan, T.M. A meta-synthesis on parenting a child with autism. Neuropsychiatr. Dis. Treat. 2016, 12, 745-762. [PubMed]

40. Osborne, L.A.; McHugh, L.; Saunders, J.; Reed, P. Parenting stress reduces the effectiveness of early teaching interventions for autistic spectrum disorders. J. Autism Dev. Disord. 2008, 38, 1092-1103. [CrossRef] [PubMed]

41. McInnes, E.; Wimpenny, P. Using qualitative assessment and review instrument software to synthesise studies on older people's views and experiences of fall prevention. Int. J. Evid. Based Healthc. 2008, 6, 337-344. [CrossRef] [PubMed]

42. Dixon-Woods, M.; Bonas, S.; Booth, A.; Jones, D.R.; Miller, T.; Sutton, A.J.; Shaw, R.L.; Smith, J.A.; Young, B. How can systematic reviews incorporate qualitative research? A critical perspective. Qual. Res. 2006, 6, 27-44. [CrossRef]

43. World Health Organization. Mental Health: A State of Well-Being. Available online: http://www.who.int/ features/factfiles/mental_health/en/ (accessed on 20 September 2017).

44. Flemming, K. Synthesis of quantitative and qualitative research: An example using Critical Interpretive Synthesis. J. Adv. Nurs. 2009, 66, 201-217. [CrossRef] [PubMed]

45. Hawker, S.; Payne, S.; Kerr, C.; Hardy, M.; Powell, J. Appraising the evidence reviewing disparate data systematically. Qual. Health Res. 2002, 12, 1284-1299. [CrossRef] [PubMed] 
46. Bitsika, V.; Sharpley, C. Development and testing of the effects of support groups on the well-being of parents of children with autism-II: Specific stress management techniques. J. Appl. Health Behav. 2000, 2, 8-15.

47. Zung, W. A rating instrument for anxiety disorders. Psychosomatics 1973, XII, 371-379. [CrossRef]

48. Zung, W. A self-rating depression scale. Arch. Gen. Psychiatry 1965, 12, 63-70. [CrossRef] [PubMed]

49. Blackledge, J.T.; Hayes, S.C. Using acceptance and commitment training in the support of parents of children diagnosed with autism. Child Fam. Behav. Ther. 2006, 28, 1-18. [CrossRef]

50. Hayes, S.C.; Strosahl, K.D.; Wilson, K.G.; Bissett, R.T.; Pistorello, J.; Toarmino, D.; Polusny, M.A.; Dykstra, T.A.; Batten, S.V.; Bergan, J.; et al. Measuring experimental avoidance: A preliminary test of a working model. Psychol. Rec. 2004, 54, 553-578. [CrossRef]

51. Hollon, S.D.; Kendall, P.C. Cognitive self-statements in depression: Development of an Automatic Thoughts Questionnaire. Cogn. Ther. Res. 1980, 4, 383-395. [CrossRef]

52. Beck, A.T.; Steer, R.A.; Brown, G.K. Manual for the Beck Depression Inventory-II; Psychological Corporation: San Antonio, TX, USA, 1996.

53. Goldbert, D.P. Manual of the General Health Questionnaire; National Foundation for Educational Research: Windsor, UK, 1978.

54. Campis, L.K.; Lyman, R.D.; Prentice-Dunn, S. The Parental Locus of Control Scale: Development and validation. J. Clin. Child Psychiatry 1986, 15, 260-267. [CrossRef]

55. Derogatis, L.R.; Melisaratos, N. The Brief Symptom Inventory: An introductory report. Psychol. Med. 1983, 13, 596-605. [CrossRef]

56. Clifford, T.; Minnes, P. Logging on: Evaluating an online support group for parents of children with autism spectrum disorders. J. Autism Dev. Disord. 2013, 43, 1662-1675. [CrossRef] [PubMed]

57. Spielberger, C.D. Manual for the State-Trait Anxiety Inventory; Consulting Psychologists Press: Palo Alto, CA, USA, 1983.

58. Spielberger, C.D.; Ritterband, L.M.; Reheiser, E.S.; Brunner, T.M. The nature and measurement of depression. Int. J. Clin. Health Psychol. 2003, 3, 209-234.

59. Behr, S.K.; Murphy, D.L.; Summers, J.A. User's Manual: Kansas Inventory of Parental Perceptions (KIPP); Beach Center on Families and Disability, University of Kansas: Lawrence, KS, USA, 1992.

60. Nachshen, J.; Woodford, L.; Minnes, P. The family stress and coping interview for families of individuals with developmental disabilities: A lifespan perspective on family adjustment. J. Intellect. Disabil. Res. 2003, 47, 285-290. [CrossRef] [PubMed]

61. Ergüner-Tekinalp, B.; Akkök, F. The effects of a coping skills training program on the coping skills, hopelessness, and stress levels of mothers of children with autism. Int. J. Adv. Couns. 2004, 26, 257-269. [CrossRef]

62. Amirkhan, J.H. A factor analytically derived measure of coping: The coping strategy indicator. J. Personal. Soc. Psychol. 1990, 59, 1066-1074. [CrossRef]

63. Beck, A.T.; Lester, L.; Tresler, L. The Hopelessness Scale. J. Consult. Clin. Psychol. 1974, 42, 861-874. [CrossRef] [PubMed]

64. Holroyd, J. The questionnaire on resources and stress: An instrument to measure family response to a handicapped family member. J. Community Psychol. 1987, 2, 92-94. [CrossRef]

65. Farmer, J.; Reupert, A. Understanding autism and understanding my child with autism: An evaluation of a group parent education program in rural Australia. Aust. J. Rural Health 2013, 21, 20-27. [CrossRef] [PubMed]

66. Feinberg, E.; Augustyn, M.; Fitzgerald, E.; Sandler, J.; Suarez, Z.F.; Chen, N.; Cabral, H.; Beardslee, W.; Silverstein, M. Improving maternal mental health after a child's diagnosis of autism spectrum disorder: Results from a randomized clinical trial. JAMA Pediatr. 2014, 168, 40-46. [CrossRef] [PubMed]

67. Carver, C.S. You want to measure coping but your protocol's too long. Int. J. Behav. Med. 1997, 4, 92-100. [CrossRef] [PubMed]

68. Rush, A.J.; Trivedi, M.H.; Ibrahim, H.M.; Carmody, T.J.; Arnow, B.; Klein, D.N.; Markowitz, J.C.; Ninan, P.T.; Kornstein, S.; Manber, R.; et al. The 16-item Quick Inventory of Depressive Symptomology (QIDS), clinical rating (QIDS-C), and self-report (QIDS-SR): A psychometric evaluation in patients with chronic major depression. Biol. Psychiatry 2003, 54, 573-583. [CrossRef]

69. Abidin, R.R. Parenting Stress Index Professional Manual, 4th ed.; Psychological Assessment Resources: Lutz, FL, USA, 2012. 
70. Ferraioli, S.; Harris, S.L. Comparative effects of mindfulness and skills-based parent training programs for parents of children with autism: Feasibility and preliminary outcome data. Mindfulness 2012, 4, 89-101. [CrossRef]

71. Brown, K.W.; Ryan, R.M. The benefits of being present: Mindfulness and its role in psychological well-being. J. Personal. Soc. Psychol. 2003, 84, 822-848. [CrossRef]

72. Giarelli, E.; Bloch, J.; Souders, M.; Levy, S.E.; Pinto-Martin, J. Intervention pilot for parents of children with autism spectrum disorder. Pediatr. Nurs. 2005, 31, 389-399. [PubMed]

73. Cohen, S.; Williamson, G.M. Perceived stress in probability sample of the United States. In The Social Psychology of Health; Spacapan, S., Oscamp, E., Eds.; Sage: Newbury Park, CA, USA, 1988; pp. 307-326.

74. Zilberg, N.; Weiss, D.; Horowitz, M. Impact of events scale: A cross validation study and some empirical evidence supporting a conceptual model of stress response syndromes. J. Consult. Clin. Psychol. 1982, 50, 407-414. [CrossRef] [PubMed]

75. Gika, D.M.; Artemiadis, A.K.; Alexopoulos, E.C.; Darviri, C.; Papanikolaou, K.; Chrousos, G.P. Use of a relaxation technique by mothers of children with autism: A case-series study. Psychol. Rep. 2012, 111, 797-804. [CrossRef] [PubMed]

76. Cohen, S.; Kamarck, T.; Mermelstein, R. A global measure of perceived stress. J. Health Soc. Behav. 1983, 24, 385-396. [CrossRef] [PubMed]

77. Izadi-Mazidi, M.; Riahi, F.; Khajeddin, H. Effect of cognitive behavior group therapy on parenting stress in mothers of children with autism. Iran J. Psychiatry Behav. Sci. 2015, 9. [CrossRef] [PubMed]

78. Jamison, J.M.; Fourie, E.; Siper, P.M.; Trelles, M.P.; George-Jones, J.; Grice, A.B.; Krata, J.; Holl, E.; Shaoul, J.; Hernandez, B.; et al. Examining the efficacy of a family peer advocate model for Black and Hispanic caregivers of children with autism spectrum disorder. J. Autism Dev. Disord. 2017, 47, 1314-1322. [CrossRef] [PubMed]

79. Brannan, A.M.; Heflinger, C.A.; Brickman, L. The caregiver strain questionnaire: Measuring the impact on the family of living with a child with serious emotional disturbance. J. Emot. Behav. Disord. 1997, 5, $212-222$. [CrossRef]

80. Koren, P.D.; DeChillo, N.; Friesen, B.J. Measuring empowerment in families whose children have emotional disabilities: A brief questionnaire. Rehabil. Psychol. 1992, 37, 305-321. [CrossRef]

81. Sherbourne, C.D.; Stewart, A.L. The MOS social support survey. Soc. Sci. Med. 1991, 32, 704-714. [CrossRef]

82. Ji, B.; Sun, M.; Yi, R.; Tang, S. Multidisciplinary parent education for caregivers of children with autism spectrum disorders. Arch. Psychiatr. Nurs. 2014, 28, 319-326. [CrossRef] [PubMed]

83. Novak, M.; Guest, C. Application of a multidimensional caregiver burden inventory. Gerontologist 1989, 29, 798-803. [CrossRef] [PubMed]

84. Wang, X.D.; Wang, X.L.; Ma, H. Rating Scales for Mental Health; Chinese Mental Health Journal Press: Beijing, China, 2009; pp. 187-190.

85. Epstein, N.B.; Baldwin, L.; Bishop, D.S. The McMaster family assessment device. J. Marital. Fam. Ther. 1983, 9, 171-180. [CrossRef]

86. General Health: 36-Item Short Form Health Survey (SF-36). Rand: Santa Monica, CA, USA. Available online: https:/ / www.rand.org/health/surveys_tools/mos/36-item-short-form/survey-instrument.html (accessed on 13 February 2018).

87. Schwarzer, R.; Jerusalem, M. Generalized Self-Efficacy scale. In Measures in Health Psychology: A User's Portfolio. Casual and Control Beliefs; Weinman, J., Wright, S., Johnston, M., Eds.; NFER-Nelson: Windsor, UK, 1994; pp. 35-37.

88. Zimet, G.D.; Powell, S.S.; Farley, G.K.; Werkman, S.; Berkoff, K.A. Psychometric characteristics of the Multidimensional Scale of Perceived Social Support. J. Personal. Assess. 1990, 55, 610-617. [CrossRef] [PubMed]

89. Joekar, S.; Farid, A.A.; Birashk, B.; Gharraee, B.; Mohammadian, M. Effectiveness of acceptance and commitment therapy in the support of parents of children with high-functioning autism. Int. J. Humanit. Cult. Stud. 2016, 2, 2763-2772.

90. Bond, F.W.; Hayes, S.C.; Baer, R.A.; Carpenter, K.M.; Orcutt, H.K.; Waltz, T.; Zettle, R.D. Preliminary psychometric properties of the Acceptance and Action Questionnaire-II: A revised measure of psychological inflexibility and experiential avoidance. Behav. Ther. 2011, 42, 676-688. [CrossRef] [PubMed] 
91. Ware, J.E., Jr.; Kosinski, M.; Keller, S.D. A 12-item short form health survey: Construction of scales and preliminary tests of reliability and validity. Med. Care 1996, 34, 220-233. [CrossRef] [PubMed]

92. Kim, J. Effects of Buddhist Ontology Focused (BOF) meditation: Pilot studies with mothers of children with developmental disabilities on their EEG and psychological well-beings. Asia Pac. J. Couns. Psychother. 2016, 7,82-100. [CrossRef]

93. Watson, D.; Clark, L.A.; Tellegen, A. Development and validation of brief measures of positive and negative affect: The PANAS scales. J. Personal. Soc. Psychol. 1988, 54, 1063-1070. [CrossRef]

94. Lovibond, S.H.; Lovibond, P.F. Manual for the Depression Anxiety Stress Scales, 2nd ed.; Psychological Foundation: Sydney, Australia, 1995; ISBN 7334-1423-0.

95. Cartwright-Hatton, S.; Wells, A. Belifs about worry and intrusions: The meta-cognitions questionnaire and its correlates. J. Anxiety Disord. 1997, 11, 279-296. [CrossRef]

96. Lovell, B.; Moss, M.; Wetherell, M.A. Assessing the feasibility and efficacy of written benefit-finding for caregivers of children with autism: A pilot study. J. Fam. Stud. 2016, 22, 32-42. [CrossRef]

97. Zigmond, A.S.; Snaith, R.P. The hospital anxiety and depression scale. Acta Psychiatr. Scand. 1983, 67, 361-370. [CrossRef] [PubMed]

98. Nguyen, C.T.; Fairclough, D.L.; Noll, R.B. Problem-Solving skills training for mothers of children recently diagnosed with autism spectrum disorder: A pilot feasibility study. Autism 2016, 20, 55-64. [CrossRef] [PubMed]

99. McNair, D.M.; Lorr, M.; Droppleman, L.F. Revised Manual for the Profile of Moods States; Educational and Industrial Testing Services: San Diego, CA, USA, 1992.

100. Creamer, M.; Bell, R.; Failla, S. Psychometric properties of the impact of event scale-Revised. Behav. Res. Ther. 2003, 41, 1489-1496. [CrossRef] [PubMed]

101. Niinomi, K.; Asano, J.; Kadoma, A.; Yoshida, K.; Ohashi, Y.; Furuzawa, A.; Yamamoto, M.; Yamakita, N.; Mori, A. Developing the "Skippu-Mama" program for mothers of children with autism spectrum disorder. Nurs. Health Sci. 2016, 18, 283-291. [CrossRef] [PubMed]

102. The WHOQOL Group. The World Health Organization Quality of Life assessment (WHOQOL): Position paper from the World Health Organization. Soc. Sci. Med. 1995, 41, 1403-1409.

103. Patra, S.; Arun, P.; Chavan, B.S. Impact of psychoeducation intervention module on parents of children with autism spectrum disorders: A preliminary study. J. Neurosci. Rural Pract. 2015, 6, 529-535. [CrossRef] [PubMed]

104. Girimaji, S.C.; Srinath, S.; Seshadri, D.K.; Krishna, D.K. Family interview for stress and coping in mental retardation (FISC-MR): A tool to study stress and coping in families of children with mental retardation. Indian J. Psychiatry 1999, 41, 341-349. [PubMed]

105. Rayan, A.; Ahmad, M. Effectiveness of mindfulness-based interventions on quality of life and positive reappraisal coping among parents of children with autism spectrum disorder. Res. Dev. Disabil. 2016, 55, 185-196. [CrossRef] [PubMed]

106. Gamefski, N.; Kraaij, V.; Spinhoven, P. Manual for the Use of the Cognitive Emotion Regulation Questionnaire; DATEC: Leiderdorp, The Netherlands, 2002.

107. WHOQOL Group. Development of the World Health Organization WHOQOL-BREF quality of life assessment. Psychol. Med. 1998, 28, 551-558.

108. Ruiz-Robledillo, N.; Sariñana-González, P.; Pérez-Blasco, J.; González-Bono, E.; Moya-Albiol, L. A mindfulness-based program improves health in caregivers of people with autism spectrum disorder: A pilot study. Mindfulness 2015, 6, 767-777. [CrossRef]

109. Miguel-Tobal, J.J.; Casado, M.I.; Cano-Vindel, A.; Spielberger, C.D. Inventario de Expression de Ira Estado-Raso: STAXI-2; TEA Ediciones: Madrid, Spain, 2001.

110. Zarit, S.H.; Reever, K.E.; Bach-Peterson, J. Relatives of the impaired elderly: Correlates of feelings of burden. Gerontologist 1980, 20, 649-654. [CrossRef] [PubMed]

111. Beck, A.T.; Steer, R.A. Beck Depression Inventory Manual; The Psychological Corporation: San Antonio, TX, USA, 1993.

112. Sandin, B.; Chorot, P. Escala de Sintomas Somáticos-Revisada (ESS-R); Universidad Nacional de Educación a Distancia (UNED): Madrid, Spain, 1995. 
113. Samadi, S.A.; McConkey, R.; Kelly, G. Enhancing parental well-being and coping through a family-centered short course for Iranian parents of children with an autism spectrum disorder. Autism 2012, 17, $27-43$. [CrossRef] [PubMed]

114. Roger, D.; Jervis, G.; Najarian, B. Detachment and coping: The construction and validation of a new scale for measuring coping strategies. Personal. Individ. Differ. 1993, 15, 619-626. [CrossRef]

115. McConkey, R.; Samadi, S.A. The impact of mutual support on Iranian parents of children with an autism spectrum disorder: A longitudinal study. Disabil. Rehabil. 2013, 35, 775-784. [CrossRef] [PubMed]

116. Tonge, B.; Brereton, A.; Kiomall, M.; Mackinnon, A.; King, N.; Rinehart, N. Effects on parental mental health of an education and skills training program for parents of young children with autism: A randomized controlled trial. J. Am. Acad. Child Adolesc. Psychiatry 2006, 45, 561-569. [CrossRef] [PubMed]

117. Zhang, W.; Yan, T.; Du, Y.; Liu, X. Brief report: Effects of solution-focused brief therapy group-work on promoting post-traumatic growth of mothers who have a child with ASD. J. Autism Dev. Disord. 2014, 44, 2052-2056. [CrossRef] [PubMed]

118. Tedeschi, R.G.; Calhoun, L.G. The posttaumatic growth inventory: Measuring the positive legacy of trauma. J. Trauma. Stress 1996, 9, 455-471. [CrossRef] [PubMed]

(C) 2018 by the authors. Licensee MDPI, Basel, Switzerland. This article is an open access article distributed under the terms and conditions of the Creative Commons Attribution (CC BY) license (http://creativecommons.org/licenses/by/4.0/). 